This proves $x y \in A$ and similarly $y x \in A$. Since $I_{f}$ is obviously closed under addition, it is a two sided ideal. An application of Lemma 3 proves that $A$ is commutative.

\title{
BIBLIOGRAPHY
}

1. N. Jacobson and C. E. Rickart, Jordan homomorphism of rings, Trans. Amer. Math. Soc. vol. 69 (1950) pp. 479-502.

2. J. L. Kelley and R. L. Vaught, The positive cone in Banach algebras, Trans. Amer. Math. Soc. vol. 74 (1953) pp. 44-55.

3. M. Krein and S. Krein, On an inner characteristic of the set of all continuous functions defined on a bicompact Hausdorff space, Comptes Rendus (Doklady) de l'Academie des Sciences de l'URSS. N. S. vol. 27 (1940) pp. 427-430.

4. S. Sherman, Order in operator algebras, Amer. J. Math. vol. 73 (1951) pp. 227232.

University of California at Los Angeles

\section{A NOTE ON VALUED LINEAR SPACES}

PAUL CONRAD

Banaschewski [1] has given a simple and elegant proof of Hahn's embedding theorem for ordered abelian groups. His method can be used to prove the author's generalization of Hahn's theorem [2, p. 11]. In this note we make use of Banaschewski's method to prove a special case of the author's theorem (which is also a generalization of Hahn's theorem) that has been proven by Gravett [3].

Let $(L, \Delta, d)$ be a valued linear space [3]. That is, $L$ is a vector space over a division ring $K, \Delta$ is a linearly ordered set with minimum element $\theta$, and $d$ is a mapping of $L$ onto $\Delta$ such that for all $x, y \in L$, $d(x)=\theta$ if and only if $x=0, d(x)=d(k x)$ for all $0 \neq k \in K$, and $d(x+y)$ $\leqq \operatorname{Max}[d(x), d(y)]$. For each $\delta \in \Delta$, let $C^{\delta}=\{x \in L: d(x) \leqq \delta\}$ and let $\bar{C}_{\delta}=\{x \in L: d(x)<\delta\}$. Let $W$ be the vector space of all mappings $f$ of $\Delta$ into the join of the spaces $C^{\delta} / C_{\delta}$ for which $f(\delta) \in C^{\delta} / C_{\delta}$ and $R_{f}=\left\{\delta \in \Delta: f(\delta) \neq C_{\delta}\right\}$ is an inversely well ordered set. $W$ is a subspace of the unrestricted direct sum $V$ of the $C^{\delta} / C_{\delta}$. W is also a valued linear space $\left(W, \Delta, d^{\prime}\right)$, with $d^{\prime}(f)$ the largest $\delta \in R(f)$.

Received by the editors February 1, 1958. 
Theorem. There exists an isomorphism $x \rightarrow \bar{x}$ of $L$ into $W$. Moreover, if $d(x)=\alpha$, then $\bar{x}(\alpha)=C_{\alpha}+x$ and $\bar{x}(\delta)=C_{\delta}$ for all $\alpha<\delta \in \Delta$. Thus this isomorphism is value-preserving.

Proof. Let $\mathfrak{S}$ be the set of all subspaces of $L$. There exists [1, Lemma 4, p. 431] a mapping $\pi$ of $\subseteq$ into $\subseteq$ such that for all $C, D \in \subseteq$, $C \oplus \pi(C)=L$ and if $C \subseteq D$, then $\pi(C) \supseteq \pi(D)$. For $x \in L$ and $\delta \in \Delta$, let $\bar{x}(\delta)=C_{\delta}+x_{\delta}$, where $L=C^{\delta} \oplus \pi\left(C^{\delta}\right)$ and $x_{\delta}$ is the component of $x$ in $C^{\delta}$. Then $\bar{x} \in V$ and it follows easily that the mapping $x \rightarrow \bar{x}$ is an isomorphism of $L$ into $V$. If $\alpha=d(x)<\delta$, then $x \in C^{\alpha} \subseteq C_{\delta}$, hence $\bar{x}(\alpha)=C_{\alpha}+x$ and $\bar{x}(\delta)=C_{\delta}$. To complete the proof it suffices to show that $R_{\tilde{x}}$ is an inversely well ordered set (for each $0 \neq x$ in $L$ ). Let $\Gamma$ be a nonempty subset of $R_{\bar{x}}$, and let $C$ be the join of the $C^{\gamma}$ for all $\gamma$ in $\Gamma . L=\pi(C) \oplus C$, and $x=y+z$ where $y \in \pi(C)$ and $z \in C . d(z)$ is the greatest element in $\Gamma$. For if $d(z)<\gamma \in \Gamma$, then $\bar{z}(\gamma)=C_{\gamma}$, and since $\pi\left(C^{\gamma}\right) \supseteq \pi(C), \bar{y}(\gamma)=C_{\gamma}$. But then $C_{\gamma}=\bar{y}(\gamma)+\bar{z}(\gamma)=\bar{x}(\gamma) \neq C_{\gamma}$.

If, as in [2], $\Delta$ is partially ordered and $d$ is multiple valued, then practically the same proof gives the embedding theorem $[2$, p. 11].

\section{REFERENCES}

1. Von Bernhard Banaschewski, Totalgeordnete Moduln, Arch. Math. vol. 7 (1956) pp. 430-440.

2. P. Conrad, Embedding theorems for abelian groups with valuations, Amer. J. Math. vol. 75 (1953) pp. 1-29.

3. K. A. H. Gravett, Valued linear spaces, Quart J. Math. Oxford Ser. (2) vol. 6 (1955) pp. 309-315.

\section{TUlane University}

